\title{
Stochastic area distributions: optimal trajectories, Maslov indices and asymptotic results
}

\author{
D.C. Khandekar \\ Theoretical Physics Division, Bhabha Atomic Research Centre, Bombay 400085 , India \\ and \\ F.W. Wiegel \\ Center for Theoretical Physics, Twente University, Enschede 7500 AE, The Netherlands
}

Received 10 November 1992; accepted for publication 16 March 1993

Communicated by A.R. Bishop

\begin{abstract}
In this paper we study the semi-classical approximation for the distribution of area associated with (i) planar polymer rings constrained to enclose a fixed algebraic area and (ii) planar rings subject to an external electric field and constrained to enclose a fixed algebraic area. We demonstrate that the results are accurate in the asymptotic regime. Moreover, we also show that in case (i) it is possible to reconstruct the exact expression for the distribution, provided the contributions from all optimal trajectories are taken into account, as well as the proper Maslov indices.
\end{abstract}

\section{Introduction}

Topologically constrained path integrals appear naturally in the description of the properties of entangled polymers. Several models to describe the entanglement effects have been proposed [1]. Amongst them is the model due to Brereton and Butler [2] which was subsequently simplified by Khandekar and Wiegel (KW) [3]. In the KW scheme the entanglement and the topological effects are incorporated by constraining the planar polymer chains to enclose a fixed algebraic area $(A)$. The properties of such polymer systems were subsequently studied in full detail by the present authors.

In most practical situations it is sufficient to know the nature of a path integral only in some asymptotic regime. This is the domain where the so called "semiclassical approximations" are expected to yield good results. While the semi-classical analysis has been well developed and applied to evaluate unconstrained path integrals [5], very little attention has been paid to the evaluation of topologically constrained path integrals. Thus it is desirable to develop a semi-clas- sical approximation for the evaluation of topologically constrained path integrals for some problems for which exact results are known, and to compare them against each other. This may perhaps suggest new ways to use this scheme for more complex problems. This is the motivation behind the present note.

We consider two cases, viz. (i) non-interacting planar polymer configurations (rings) constrained to enclose a fixed algebraic area, and (ii) planar polymer rings, subject to an external electric field, and constrained to enclose a fixed algebraic area. The area distributions for these cases have been derived by the present authors in three earlier papers $[3,4,6]$. In this note we obtain the "semi-classical" approximations to the distributions of the enclosed area. We show that the expressions correspond to the asymptotic limit (a phrase to be made more precise later) of the exact distributions. For the case of non-interacting configurations we pursue our investigations further and demonstrate that, if the contributions from all optimal trajectories are taken into account, the result reconstructs the exact expression for the distribution function. 


\section{Non-interacting planar system}

The Lagrangian $\mathscr{L}$ for a non-interacting planar polymer configuration $\boldsymbol{r}(\nu) \equiv(x, y)$ can be written as

$\mathscr{L}=\frac{\dot{\boldsymbol{r}}^{2}}{l^{2}}$,

where $l$ is related to the mean bond length between monomers and where the dot denotes $\partial / \partial \nu$. Next we impose the constraint that the configurations enclose a fixed algebraic area

$A=\frac{1}{2} \int_{0}^{N}(x \dot{y}-y \dot{x}) \mathrm{d} \nu$,

$N$ being the total number of monomers. The constraint is incorporated into (1) by a Lagrange multiplier $\lambda$, so we write the modified Lagrangian as

$\mathscr{L}=\frac{\dot{r}^{2}}{l^{2}}-\lambda(x \dot{y}-y \dot{x})$.

The optimal trajectory for the Lagrangian (3) is described by the Euler-Lagrange equations

$\ddot{x}+\omega \dot{y}=0, \quad \ddot{y}-\omega \dot{x}=0$,

subject to the boundary conditions $x(0)=x^{\prime}$, $y(0)=y^{\prime}, x(N)=x^{\prime \prime}$ and $y(N)=y^{\prime \prime}$. Moreover, the quantity $\omega$ denotes $\frac{1}{2} \lambda l^{2}$. The set (4) is easy to solve and we obtain

$x=A_{+} \cos (\omega \nu)-A_{-} \cos [(N-\nu) \omega]$

$-C_{2} / \omega$

$y=A_{+} \sin (\omega \nu)+A_{-} \sin [\omega(N-\nu)]$

$+C_{1} / \omega$,

where $A_{ \pm}$and $C_{1}, C_{2}$ are constants which can be determined using the boundary conditions; they are found to be given by

$A_{ \pm}=\frac{1}{2}\left(\frac{X}{\cos (\omega N)-1} \pm \frac{Y}{\sin (\omega N)}\right)$,

$C_{1}=\omega\left[y^{\prime \prime}-A_{+} \sin (\omega N)\right]$,

$C_{2}=\omega\left[A_{+} \cos (\omega N)-A_{-}-x^{\prime \prime}\right]$,

with $X=x^{\prime \prime}-x^{\prime}$ and $Y=y^{\prime \prime}-y^{\prime}$.

Using expression (5) for the optimal trajectory we now evaluate the action functional along the classical trajectory. It reads as

$$
\begin{aligned}
S_{\mathrm{cl}} & =\frac{2 \omega A}{l^{2}}+\frac{\omega}{l^{2}}\left[x^{\prime \prime} y^{\prime}-y^{\prime \prime} x^{\prime}\right. \\
& \left.+\frac{1}{2} \cot \left(\frac{1}{2} \omega N\right)\left(X^{2}+Y^{2}\right)\right] .
\end{aligned}
$$

We still have to determine the Lagrange parameter $\lambda(\omega)$. This is done by demanding that

$A=\frac{1}{2} \int_{0}^{N}(x \dot{y}-y \dot{x}) \mathrm{d} \nu$.

This implies

$$
\begin{aligned}
A= & \frac{1}{2 \omega}\left\{-\left(X C_{1}+Y C_{2}\right)\right. \\
& \left.+\omega^{2} N\left[A_{+}^{2}+A_{-}^{2}-2 A_{+} A_{-} \cos (\omega N)\right]\right\} .
\end{aligned}
$$

We now concentrate on closed polymer rings. As $x^{\prime \prime} \rightarrow x^{\prime}$ and $y^{\prime \prime} \rightarrow y^{\prime}$ it is easy to see that $A_{ \pm}, C_{1}$ and $C_{2} \rightarrow 0$. Hence, in order to have a non-zero value for $A_{ \pm}$, and thus for the area $A$, it follows that $\omega$ has to have one of the values

$\omega_{n}=2 n \pi / N, \quad n= \pm 1, \pm 2, \ldots$.

We do not allow the solution $n=0$ since this implies the absence of the area constraint. We now use the well known expression for the semi-classical approximation for the propagator $G\left(x^{\prime \prime}, N \mid x^{\prime}, 0\right)$, namely

$G\left(x^{\prime \prime}, N \mid x^{\prime}, 0\right) \approx \exp \left(-S_{\mathrm{cl}}\right)$.

Retaining only the root $n=1$, we first observe that the normalizability of $G$ implies that $A>0$ for $n>0$ and $A<0$ for $n<0$. Hence we have

$$
\begin{aligned}
& P(A)=G\left(x^{\prime}, N \mid x^{\prime}, 0\right) \\
& \quad \approx C \exp \left(-4 \pi|A| / l^{2} N\right) .
\end{aligned}
$$

The normalization constant $C$ can be trivially found and the complete expression for the semi-classical distribution of the area reads as

$P(A)=\frac{2 \pi}{N l^{2}} \exp \left(-4 \pi|A| / N l^{2}\right)$.

We compare this with the cxact expression for $P(A)$ derived by the authors in ref. [3]:

$P(A)=\left[2 N l^{2} \cosh ^{2}\left(2 \pi A / N l^{2}\right)\right]^{-1}$. 
Expand the $\cosh ^{2}\left(2 \pi A / N l^{2}\right)$ in powers of $\exp \left(-4 \pi A / N l^{2}\right)$. One finds that the first term of the series is identical to (12a). This suggests that, if the constraint is properly accounted for in the optimal trajectory, the resulting expression for the path integral is accurate in the asymptotic regime $\left(A / N l^{2} \gg 1\right)$.

We shall now take this analysis further by asking whether the approximation can be improved. A naive attitude would be to add all contributions $\exp \left(-S_{n}\right), S_{n}$ being the value of the action functional (7) with $\omega=\omega_{n}$. Here we face difficulties. If we were to add these contributions with equal weights we would find

$$
\begin{aligned}
& P(A)=\sum_{n=1}^{\infty} \exp \left(-S_{n}\right)=\sum_{n=1}^{\infty} \exp \left(-4 \pi n A / N l^{2}\right) \\
& =\frac{\exp \left(-4 \pi A / N l^{2}\right)}{1-\exp \left(-4 \pi A / N l^{2}\right)}
\end{aligned}
$$

which is wrong! It is easy to see that this formula for $P(A)$ is not even normalizable and hence cannot qualify for a probability distribution. In such a circumstance we again seek guidance for the procedure from the case in which there is no constraint. The complete semi-classical approximation to the propagator is given by

$$
\begin{aligned}
& G\left(x^{\prime \prime}, N \mid x^{\prime}, 0\right)=\frac{1}{(2 \pi)^{d / 2}} \sum_{n}\left[\exp \left(\frac{1}{2} \mathrm{i} \pi\right)\right]^{k(n)} \\
& \quad \times\left|\frac{\partial^{2} S}{\partial r^{\prime \prime} \partial r^{\prime}}\right|^{d / 2} \exp \left(-S_{n}\right),
\end{aligned}
$$

where $d$ is the dimensionality of the system and where $k(n)$ is the Maslov index associated with the $n$th trajectory. Also, $\left|\partial^{2} S / \partial r^{\prime \prime} \partial r^{\prime}\right|$ is the van Vleck determinant (cf. ref. [5]).

The evaluation of the van Vleck determinant is the first step. As $x^{\prime} \rightarrow x^{\prime \prime}$ and $y^{\prime} \rightarrow y^{\prime \prime} S_{\mathrm{cl}}$ and $A$ go to zero for a fixed value of $\omega N \neq 2 n \pi$. Therefore, we have to evaluate the limiting behaviour of these quantities as the polymer ring is formed. It is easy to verify from eq. (6) that for $\omega_{n}=2 n \pi / N+\delta$, the algebraic area $A$ has the expression

$$
A=n \pi \frac{X^{2}+Y^{2}}{\delta^{2}}+\mathrm{O}(\delta),
$$

which implies that for a finite algebraic area $A$ the quantity $\left(X^{2}+Y^{2}\right) / \delta^{2}$ should tend to a finite limit as $\delta \rightarrow 0$. This yields

$C_{1} X+C_{2} Y=\omega\left(x^{\prime \prime} y^{\prime}-y^{\prime \prime} x^{\prime}\right)+\mathrm{O}(\delta)$.

Substituting these expressions into (7) we arrive at the result

$S_{n}=\frac{2 \omega_{n} A+\omega\left(x^{\prime \prime} y^{\prime}-y^{\prime \prime} x^{\prime}\right)}{l^{2}}+\mathrm{O}(\delta)$.

Hence, in the limit $\delta \rightarrow 0$, the van Vleck determinant reads as

$$
\begin{gathered}
\left|\frac{\partial^{2} S}{\partial \boldsymbol{r}^{\prime \prime} \partial \boldsymbol{r}^{\prime}}\right|=\left|\begin{array}{cc}
0 & -\omega_{n} / l^{2} \\
\omega_{n} / l^{2} & 0
\end{array}\right| \\
=\left(\frac{\omega_{n}}{l^{2}}\right)^{2}=\left(\frac{2 n \pi}{N l^{2}}\right)^{2} .
\end{gathered}
$$

Next, we consider expression (17) for $S_{\mathrm{cl}}$. For closed rings $x^{\prime \prime} \rightarrow x^{\prime}, y^{\prime \prime} \rightarrow y^{\prime}$. Therefore

$S_{n}=\frac{2 \omega_{n} A}{l^{2}}=\frac{4 n \pi A}{N l^{2}}$.

Finally, we must evaluate $k(n)$. For a given $r^{\prime}$ and $\boldsymbol{r}^{\prime \prime}\left(\boldsymbol{r}^{\prime} \neq \boldsymbol{r}^{\prime \prime}\right)$ it easy to see from the expression for $S$ that $\omega_{n}=2 n \pi$ are the singular points both of $S$ and of the van Vleck determinant. This implies $k(n)=2 n-2$. Therefore, the complete expression for the distribution of the area reads as

$$
\begin{aligned}
& P(A)=\frac{1}{\pi} \sum_{n=1}^{\infty}\left[\exp \left(\frac{1}{2} \mathrm{i} \pi\right)\right]^{2(n-1)} \\
& \quad \times\left(2 n \pi / N l^{2}\right) \exp \left(-4 n \pi A / N l^{2}\right),
\end{aligned}
$$

which is the exact expression for the distribution of the area.

\section{Planar configurations in an external electric field}

We proceed as in the previous section. The Lagrangian for the polymer configurations reads as

$\mathscr{L}=\frac{\dot{\boldsymbol{r}}^{2}}{l^{2}}-q \boldsymbol{E} \cdot \boldsymbol{r}$,

where $q$ is the charge on each monomer and $\boldsymbol{E}=$ $\left(E_{1}, E_{2}\right)$ is a uniform electric field. The area constraint is incorporated through a Lagrange multiplier and we define the modified Lagrangian $\mathscr{L}$ as 
$\mathscr{L}=\frac{\dot{\boldsymbol{r}}^{2}}{l^{2}}-q E \cdot \boldsymbol{r}-\lambda(x \dot{y}-y \dot{x})$.

The associated Euler-Lagrange equations can be set up easily and are given by

$\frac{2 \ddot{x}}{l^{2}}+\lambda \dot{y}+q E_{1}=0$,

$\frac{2 \ddot{y}}{l^{2}}-\lambda \dot{x}+q E_{2}=0$,

subject to boundary conditions $x(0)=x^{\prime}$, $x(N)=x^{\prime \prime}, y(0)=y^{\prime}$ and $y(N)=y^{\prime \prime}$. One can very easily solve the set (23) to arrive at

$$
\begin{aligned}
\xi= & A_{+} \cos (\omega \nu)-A_{-} \cos [\omega(N-\nu)] \\
& -C_{2} / \omega, \\
\eta= & A_{+} \sin (\omega \nu)+A_{-} \sin [(N-\nu) \omega] \\
& +C_{1} / \omega,
\end{aligned}
$$

where $\xi$ and $\eta$ are new variables which are related to $x$ and $y$ by the expressions

$x=\xi+\alpha \nu, \quad \alpha=q E_{2} / \lambda$,

$y=\eta+\beta \nu, \beta=-q E_{1} / \lambda$.

Moreover, $A_{ \pm}, C_{1}$ and $C_{2}$ are constants to be determined using the boundary conditions. They are found to be given by the expressions

$A_{ \pm}=\frac{1}{2} N\left(\frac{\alpha}{1-\cos (\omega N)} \mp \frac{\beta}{\sin (\omega N)}\right)$,

$\frac{C_{1}}{\omega}=-\frac{1}{2} N\left[\alpha \cot \left(\frac{1}{2} \omega N\right)+\beta\right]$,

$\frac{C_{2}}{\omega}=\frac{1}{2} N\left[\alpha-\beta \cot \left(\frac{1}{2} \omega N\right)\right]$.

As in section 2, we now evaluate the value of the action functional $S_{\mathrm{cl}}$ along the optimal trajectory. After some straightforward algebra one finds

$S_{\mathrm{cl}}=\frac{Q^{2} E^{2} L}{16 x^{2}}\left(\frac{x^{2}}{\sin ^{2}(x)}+2 x \cot (x)-3\right)$,

where $x=\frac{1}{2} \omega N, Q=N q, L=N l^{2}$.

We still have to determine the value of the parameter $\lambda$ or $x$. This is again done by demanding
$A=\frac{1}{2} \int_{0}^{N}(x \dot{y}-y \dot{x}) \mathrm{d} \nu ;$

it leads to a transcendental equation

$A=\frac{Q^{2} E^{2} L^{2}}{64 x^{3}}\left(\frac{x^{2}}{\sin ^{2}(x)}+x \cot (x)-2\right)$.

The last equation cannot be solved exactly. However, it is clear that it has an infinity of solutions, each in the interval $[k \pi,(k+1) \pi], k=0,1, \ldots$. In order to investigate the asymptotic behaviour of the distribution $P(A)$ of the area it suffices to consider the solution in the interval $[0, \pi]$. For very strong electric fields $A / E^{2} \rightarrow 0$. Hence the solution to (29) lies very close to zero. In this regime we can use the approximation

$\cot (x) \simeq 1 / x-\frac{1}{3} x+\frac{1}{5} x^{3}$,

$\operatorname{cosec}(x) \simeq 1 / x+\frac{1}{6} x-\frac{7}{360} x^{3}$,

which when substituted into (29) lead to

$A=\frac{Q^{2} E^{2} L^{2}}{64} \frac{x}{90}$.

When this value of $x$ is substituted into the expression for $S$ we obtain

$$
\begin{aligned}
S_{\mathrm{cl}} & =\frac{Q^{2} E^{2} L}{16 \times 30}\left(\frac{64 \times 90 A}{Q^{2} E^{2} L^{2}}\right)^{2} \\
& =\frac{a A^{2}}{E^{2}},
\end{aligned}
$$

apart from an additive constant. Therefore, the normalized expression for the distribution of the area is given by

$P(A)=\frac{1}{E} \sqrt{a / \pi} \exp \left(-a A^{2} / E^{2}\right)$,

which is the asymptotic limit of the exact expression for the distribution of $A$ as derived in ref. [6].

On the other hand, when the electric field is weak eq. (29) suggests that $x$ is very close to $\pi$. Therefore, using $x=\pi-\delta, \delta \rightarrow 0$, we get

$A=\frac{Q^{2} E^{2} L^{2}}{64 \pi \delta^{2}}$ 
When this expression is substituted into the expression for $S$, it simplifies to

$S=\frac{4 \pi A}{L}$.

This value of $S$ leads to the asymptotic result (12) for the distribution of the area in the case in which there is no external electric field.

\section{References}

[1] F.W. Wiegel, Introduction to path-integral methods in physics and polymer science (World Scientific, Singapore, 1986).

[2] M.G. Brereton and C. Butler, J. Phys. A 20 (1987) 3955.

[3] D.C. Khandekar and F.W. Wiegel, J. Phys. A 21 (1988) L563.

[4] D.C. Khandekar and F.W. Wiegel, J. Phys. (Paris) 50 (1989) 263.

[5] L.S. Schulman, Techniques and applications of path integration (Wiley, New York, 1981).

[6] F.W. Wiegel and D.C. Khandekar, Int. J. Mod. Phys. B 6 (1992) 3147. 